\title{
ON THE SONG RECURRENCE RELATION FOR THE RIEMANN ZETA FUNCTION
}

\author{
MIRCEA MERCA
}

Received 21 May, 2016

\begin{abstract}
In this paper, the author uses the generating function for the Bernoulli numbers in order to obtain a new proof for a known linear recurrence relation of the Riemann zeta function with even integer arguments, $\zeta(2 n)$.
\end{abstract}

2010 Mathematics Subject Classification: 11B68; 11B40

Keywords: Riemann zeta function, Bernoulli numbers, recurrences

\section{INTRODUCTION}

Originally the Riemann zeta function was defined for real arguments by Euler as

$$
\zeta(x)=\sum_{n=1}^{\infty} \frac{1}{n^{x}}, \quad x>1 .
$$

Euler first started to develop the theory of this function and obtained in 1734 the famous formula for even positive zeta values

$$
\zeta(2 n)=(-1)^{n+1} \frac{(2 \pi)^{2 n}}{2 \cdot(2 n) !} B_{2 n},
$$

where $n$ is a positive integer and $B_{n}$ is the $n$-th Bernoulli number. There are many proofs of this formula, some of them elementary, see, e.g., [1-3, 5, 10, 12-14].

The following recurrence relation for $\zeta(2 n)$ has been derived by Song [11, eq. 15] in 1987 using Fourier series expansion of periodic functions. In this work, Song did not make use of the Riemann zeta function and always wrote the sums explicitly.

Theorem 1. For $n>0$,

$$
(-1)^{n} \frac{\pi^{2 n} \cdot n}{(2 n+1) !}+\sum_{k=0}^{n-1}(-1)^{k} \frac{\pi^{2 k}}{(2 k+1) !} \zeta(2 n-2 k)=0 .
$$


We see that this linear recurrence relation does not require a priori knowledge of the Bernoulli numbers. If $n$ is small, then it is relatively easy to evaluate $\zeta(2 n)$, namely

$$
\begin{aligned}
& \zeta(2)=\frac{\pi^{2}}{3 !}, \\
& \zeta(4)=\frac{\pi^{2}}{3 !} \cdot \zeta(2)-\frac{\pi^{4}}{5 !} \cdot 2=\frac{\pi^{4}}{90}, \\
& \zeta(6)=\frac{\pi^{2}}{3 !} \cdot \zeta(4)-\frac{\pi^{4}}{5 !} \cdot \zeta(2)+\frac{\pi^{6}}{7 !} \cdot 3=\frac{\pi^{6}}{945}, \\
& \zeta(8)=\frac{\pi^{2}}{3 !} \cdot \zeta(6)-\frac{\pi^{4}}{5 !} \cdot \zeta(4)+\frac{\pi^{6}}{7 !} \cdot \zeta(2)-\frac{\pi^{8}}{9 !} \cdot 4=\frac{\pi^{8}}{9450} .
\end{aligned}
$$

In 2008, Lettington [7, Lemma 11.1.2, pp. 140-142] rediscovered this result in his doctoral thesis by deriving the Bernoulli recurrence relation

$$
2^{2 n-1} B_{2 n}=\frac{n}{2 n+1}-\frac{1}{2 n+1} \sum_{k=1}^{n-1}\left(\begin{array}{c}
2 n+1 \\
2 k
\end{array}\right) 2^{2 k-1} B_{2 k} .
$$

Moreover, he showed in [8] that this linear recurrence for the Riemann zeta function occurs naturally in a certain variety of odd-side magic square matrix with multiplicative symmetries. These symmetries are discussed more generally in [4].

In this paper, based on the generating function of the Bernoulli numbers and Euler's formula (1.1), we provide a proof of Theorem 1 which is different from Song's and Lettington's proofs. We remark that the proof via generating functions for the Bernoulli numbers is of interest, as can be observed in the recent paper by Coffey [6].

\section{A PROOF OF THEOREM 1}

We consider the following two series:

$$
\sum_{n=0}^{\infty} \frac{B_{n}}{n !} x^{n}=\frac{x}{e^{x}-1}, \quad|x|<2 \pi
$$

and

$$
\sum_{n=0}^{\infty} \frac{1}{n !} x^{n}=e^{x}, \quad \text { for all } x .
$$

For $|x|<\pi$, we can write

$$
\left(\sum_{n=0}^{\infty} \frac{B_{n}}{n !}(2 x)^{n}\right)\left(\sum_{n=0}^{\infty} \frac{1}{n !} x^{n}\right)=\frac{2 x e^{x}}{e^{2 x}-1}
$$


and

$$
2 \sum_{n=0}^{\infty} \frac{B_{n}}{n !} x^{n}-\sum_{n=0}^{\infty} \frac{B_{n}}{n !}(2 x)^{n}=\frac{2 x}{e^{x}-1}-\frac{2 x}{e^{2 x}-1}=\frac{2 x e^{x}}{e^{2 x}-1} .
$$

Thus we deduce that

$$
\left(\sum_{n=0}^{\infty} \frac{2^{n} B_{n}}{n !} x^{n}\right)\left(\sum_{n=0}^{\infty} \frac{1}{n !} x^{n}\right)=\sum_{n=0}^{\infty} \frac{2-2^{n}}{n !} B_{n} x^{n}, \quad|x|<\pi .
$$

Equating coefficients of $x^{2 n+1}$ on each side give the relation

$$
\sum_{k=0}^{2 n+1} \frac{2^{k} B_{k}}{k ! \cdot(2 n+1-k) !}=0
$$

Moreover, taking into account that $B_{1}=-1 / 2$ and $B_{2 n+1}=0$ for $n>0$, we deduce

$$
\frac{1}{(2 n) !}-\sum_{k=0}^{n} \frac{2^{2 k} B_{2 k}}{(2 k) ! \cdot(2 n+1-2 k) !}=0
$$

or

$$
\frac{1}{(2 n) !}-\sum_{k=0}^{n} \frac{2^{2 n-2 k} B_{2 n-2 k}}{(2 n-2 k) ! \cdot(2 k+1) !}=0
$$

Using Euler's formula (1.1), we obtain the relation

$$
2 \sum_{k=0}^{n} \frac{(-1)^{n-k+1} \zeta(2 n-2 k)}{\pi^{2 n-2 k} \cdot(2 k+1) !}-\frac{1}{(2 n) !}=0
$$

that can be rewritten as

$$
\sum_{k=0}^{n-1}(-1)^{k} \frac{\pi^{2 k}}{(2 k+1) !} \zeta(2 n-2 k)+(-1)^{n} \frac{\pi^{2 n}}{(2 n+1) !} \cdot n=0 .
$$

This completes the proof.

\section{CONCLUDING REMARKS.}

Due to the results of Euler and the more recent works described herein, we know that the linear recurrence relations for $\zeta(2 n)$ are intimately related to the properties of the Bernoulli numbers. In this paper, we have obtained a new proof of the linear recurrence for $\zeta(2 n)$ considered by Song and Lettington. Moreover, equating coefficients of $x^{2 n}$ on each side of the relation (2.1), we obtain the relation

$$
\sum_{k=0}^{n} \frac{2^{2 k} B_{2 k}}{(2 k) !(2 n-2 k) !}=\frac{2-2^{2 n}}{(2 n) !} B_{2 n}+\frac{2 n}{(2 n) !},
$$

that allows us to derive the following recurrence relation for $\zeta(2 n)$. 
Theorem 2. For $n>0$,

$$
\zeta(2 n)+\frac{2^{2 n-1}}{2^{2 n}-1}\left((-1)^{n} \frac{\pi^{2 n}}{(2 n) !} \cdot \frac{2 n-1}{2}+\sum_{k=1}^{n-1}(-1)^{k} \frac{\pi^{2 k}}{(2 k) !} \zeta(2 n-2 k)\right)=0 .
$$

According to the last theorem, we can write

$$
\begin{aligned}
& \zeta(2)=\frac{2}{3} \cdot \frac{\pi^{2}}{2 !} \cdot \frac{1}{2}=\frac{\pi^{2}}{6}, \\
& \zeta(4)=\frac{8}{15} \cdot\left(\frac{\pi^{2}}{2 !} \cdot \zeta(2)-\frac{\pi^{4}}{4 !} \cdot \frac{3}{2}\right)=\frac{\pi^{4}}{90}, \\
& \zeta(6)=\frac{32}{63} \cdot\left(\frac{\pi^{2}}{2 !} \cdot \zeta(4)-\frac{\pi^{4}}{4 !} \cdot \zeta(2)+\frac{\pi^{6}}{6 !} \cdot \frac{5}{2}\right)=\frac{\pi^{6}}{945}, \\
& \zeta(8)=\frac{128}{255} \cdot\left(\frac{\pi^{2}}{2 !} \cdot \zeta(6)-\frac{\pi^{4}}{4 !} \cdot \zeta(4)+\frac{\pi^{6}}{6 !} \cdot \zeta(2)-\frac{\pi^{8}}{8 !} \cdot \frac{7}{2}\right)=\frac{\pi^{8}}{9450} .
\end{aligned}
$$

A final remark is that as with Lemma 2.1 of [9], these type of recurrence relations often reduce to relations involving Bernoulli numbers, binomial coefficients and powers of 2. Here we find that Theorems 1 and 2 are consequences of the recurrence relation

$$
\sum_{k=0}^{n}\left(\begin{array}{l}
n \\
k
\end{array}\right) 2^{k} B_{k}=\left(2-2^{n}\right) B_{n}
$$

\section{ACKNOWLEDGEMENT}

The author likes to thank the anonymous referees for their helpful assistance. Special thanks go to Dr. Oana Merca for the careful reading of the manuscript.

\section{REFERENCES}

[1] T. M. Apostol, "Another elementary proof of Euler's formula for $\zeta(2 n)$." Amer. Math. Monthly, vol. 80, pp. 425-432, 1973, doi: 10.2307/2319093.

[2] R. Ayoub, "Euler and the Zeta function." Amer. Math. Monthly, vol. 81, pp. 1067-1086, 1974, doi: $10.2307 / 2319041$.

[3] B. C. Berndt, "Elementary evaluation of $\zeta(2 n)$." Math. Magazine, vol. 48, pp. 148-154, 1975, doi: $10.2307 / 2689696$.

[4] R. Brunnock, M. C. Lettington, and K. M. Schmidt, "On square roots and norms of matrices with symmetry properties.” Linear Algebra Appl., vol. 459, pp. 175-207, 2014, doi: 10.1016/j.1aa.2014.06.054.

[5] X. Chen, "Recursive formulas for $\zeta(2 k)$ and $L(2 k-1)$." College Math. J., vol. 26, no. 5, pp. 372-376, 1995.

[6] M. W. Coffey, "Bernoulli identities, zeta relations, determinant expressions, Mellin transforms, and representation of the Hurwitz numbers," arXiv:1601.01673.

[7] M. C. Lettington, PhD Thesis. Cardiff University, 2008.

[8] M. C. Lettington, "Fleck's congruence, associated magic squares and zeta identity." Funct. Approx. Comment. Math., vol. 45, no. 2, pp. 165-205, 2011. 
[9] M. C. Lettington, "A trio of Bernoulli relations, their implications for the Ramanujan polynomials and the special values of the Riemann zeta function," Acta Arith., vol. 158, pp. 1-31, 2013, doi: 10.4064/aa158-1-1.

[10] T. Osler, "Finding $\zeta(2 n)$ from a product of sines." Amer. Math. Monthly, vol. 111, pp. 52-54, 2014, doi: $10.2307 / 4145017$.

[11] I. Song, "A recursive formula for even order harmonic series." J. Comput. Appl. Math., vol. 21, pp. 251-256, 1988, doi: 10.1016/0377-0427(88)90274-9.

[12] H. Tsumura, "An elementary proof of Euler's formula for $\zeta(2 n)$." Amer. Math. Monthly, vol. 111, pp. 430-431, 2004, doi: 10.2307/4145270.

[13] G. T. Williams, "A new method of evaluating $\zeta(2 n) . "$ Amer. Math. Monthly, vol. 60, pp. 19-25, 1953, doi: $10.2307 / 2306473$.

[14] K. S. Williams, “On $\sum_{n=1}^{\infty}\left(1 / n^{2 k}\right)$." Math. Magazine, vol. 44, pp. 273-276, 1971, doi: $10.2307 / 2688638$.

Author's address

Mircea Merca

University of Craiova, Department of Mathematics, 13 A. I. Cuza, 200585 Craiova, Romania

E-mail address: mircea.mercaeprofinfo.edu.ro 